Article

\title{
Properties of Spiral-Like Close-to-Convex Functions Associated with Conic Domains
}

\author{
Hari M. Srivastava ${ }^{1,2}{ }^{\circledR}$, Nazar Khan ${ }^{3}$, Maslina Darus ${ }^{4}\left(\mathbb{D}\right.$, Muhammad Tariq Rahim ${ }^{3}$, \\ Qazi Zahoor Ahmad ${ }^{3, *}$ and Yousra Zeb $^{3}$ \\ 1 Department of Mathematics and Statistics, University of Victoria, Victoria, BC V8W 3R4, Canada \\ 2 Department of Medical Research, China Medical University Hospital, China Medical University, \\ Taichung 40402, Taiwan \\ 3 Department of Mathematics, Abbottabad University of Science and Technology, Abbottabad 22010, Pakistan \\ 4 School of Mathematical Sciences, Faculty of Sciences and Technology, Universiti Kebangsaan Malaysia, \\ Bangi 43600, Selangor, Malaysia \\ * Correspondence: zahoorqazi5@gmail.com; Tel.: +92-334-96-60-162
}

Received: 26 June 2019; Accepted: 29 July 2019; Published: 6 August 2019

check for updates

\begin{abstract}
In this paper, our aim is to define certain new classes of multivalently spiral-like, starlike, convex and the varied Mocanu-type functions, which are associated with conic domains. We investigate such interesting properties of each of these function classes, such as (for example) sufficiency criteria, inclusion results and integral-preserving properties.
\end{abstract}

Keywords: analytic functions; multivalent functions; starlike functions; close-to-convex functions; uniformly starlike functions; uniformly close-to-convex functions; conic domains

MSC: Primary 05A30; 30C45; Secondary 11B65; 47B38

\section{Introduction and Motivation}

Let $\mathcal{A}(p)$ denote the class of functions of the form:

$$
f(z)=z^{p}+\sum_{n=1}^{\infty} a_{n+p} z^{n+p} \quad(p \in \mathbb{N}=\{1,2,3, \cdots\}),
$$

which are analytic and $p$-valent in the open unit disk:

$$
\mathbb{E}=\{z: z \in \mathbb{C} \text { and }|z|<1\}
$$

In particular, we write:

$$
\mathcal{A}(1)=\mathcal{A} \text {. }
$$

Furthermore, by $\mathcal{S} \subset \mathcal{A}$, we shall denote the class of all functions that are univalent in $\mathbb{E}$.

The familiar class of $p$-valently starlike functions in $\mathbb{E}$ will be denoted by $\mathcal{S}^{*}(p)$, which consists of functions $f \in \mathcal{A}(p)$ that satisfy the following conditions:

$$
\Re\left(\frac{z f^{\prime}(z)}{f(z)}\right)>0 \quad(\forall z \in \mathbb{E}) .
$$

One can easily see that:

$$
\mathcal{S}^{*}(1)=\mathcal{S}^{*},
$$

where $\mathcal{S}^{*}$ is the well-known class of normalized starlike functions (see [1]). 
We denote by $\mathcal{K}$ the class of close-to-convex functions, which consists of functions $f \in \mathcal{A}$ that satisfy the following inequality:

$$
\Re\left(\frac{z f^{\prime}(z)}{g(z)}\right)>0 \quad(\forall z \in \mathbb{E})
$$

for some $g \in \mathcal{S}^{*}$.

For two functions $f$ and $g$ analytic in $\mathbb{E}$, we say that the function $f$ is subordinate to the function $g$ and write as follows:

$$
f \prec g \text { or } f(z) \prec g(z),
$$

if there exists a Schwarz function $w$, which is analytic in $\mathbb{E}$ with:

$$
w(0)=0 \quad \text { and } \quad|w(z)|<1,
$$

such that:

$$
f(z)=g(w(z)) .
$$

Furthermore, if the function $g$ is univalent in $\mathbb{E}$, then it follows that:

$$
f(z) \prec g(z) \quad(z \in \mathbb{E}) \Longrightarrow f(0)=g(0) \text { and } f(\mathbb{E}) \subset g(\mathbb{E}) .
$$

Next, for a function $f \in \mathcal{A}(p)$ given by (1) and another function $g \in \mathcal{A}(p)$ given by:

$$
g(z)=z^{p}+\sum_{n=2}^{\infty} b_{n+p} z^{n+p} \quad(\forall z \in \mathbb{E})
$$

the convolution (or the Hadamard product) of $f$ and $g$ is given by:

$$
(f * g)(z)=z^{p}+\sum_{n=2}^{\infty} a_{n+p} b_{n+p} z^{n+p}=(g * f)(z) .
$$

The subclass of $\mathcal{A}$ consisting of all analytic functions with a positive real part in $\mathbb{E}$ is denoted by $\mathcal{P}$. An analytic description of $\mathcal{P}$ is given by:

$$
h(z)=1+\sum_{n=1}^{\infty} c_{n} z^{n} \quad(\forall z \in \mathbb{E}) .
$$

Furthermore, if:

$$
\Re\{h(z)\}>\rho,
$$

then we say that $h$ is in the class $\mathcal{P}(\rho)$. Clearly, one see that:

$$
\mathcal{P}(0)=\mathcal{P} \text {. }
$$

Historically, in the year 1933, Spaček [2] introduced the $\beta$-spiral-like functions as follows.

Definition 1. A function $f \in \mathcal{A}$ is said to be in the class $\mathcal{S}^{*}(\beta)$ if and only if:

$$
\Re\left(e^{i \beta} \frac{z f^{\prime}(z)}{f(z)}\right)>0 \quad(\forall z \in \mathbb{E})
$$

for:

$$
\beta \in \mathbb{R} \text { and }|\beta|<\frac{\pi}{2}
$$

where $\mathbb{R}$ is the set of real numbers. 
In the year 1967, Libera [3] extended this definition to the class of functions, which are spiral-like of order $\rho$ denoted by $\mathcal{S}_{\rho}^{*}(\beta)$ as follows.

Definition 2. A function $f \in \mathcal{A}$ is said to be in the class $\mathcal{S}_{\rho}^{*}(\beta)$ if and only if:

$$
\begin{gathered}
\Re\left(e^{i \beta} \frac{z f^{\prime}(z)}{f(z)}\right)>\rho \quad(\forall z \in \mathbb{E}) \\
\left(0 \leqq \rho<1 ; \beta \in \mathbb{R} \quad \text { and } \quad|\beta|<\frac{\pi}{2}\right),
\end{gathered}
$$

where $\mathbb{R}$ is the set of real numbers.

The above function classes $\mathcal{S}^{*}(\beta)$ and $\mathcal{S}_{\rho}^{*}(\beta)$ have been studied and generalized by different viewpoints and perspectives. For example, in the year 1974, a subclass $S_{\beta}^{\alpha}(\rho)$ of spiral-like functions was introduced by Silvia (see [4]), who gave some remarkable properties of this function class. Subsequently, Umarani [5] defined and studied another function class $S C(\alpha, \beta)$ of spiral-like functions. Recently, Noor et al. [6] generalized the works of Silvia [4] and Umarani [5] by defining the class $M(p, \alpha, \beta, \rho)$. Here, in this paper, we define certain new subclasses of spiral-like close-to-convex functions by using the idea of Noor et al. [6] and Umarani [5].

We now recall that Kanas et al. (see [7,8]; see also [9]) defined the conic domains $\Omega_{k}(k \geqq 0)$ as follows:

$$
\Omega_{k}=\left\{u+i v: u>k \sqrt{(u-1)^{2}+v^{2}}\right\} .
$$

By using these conic domains $\Omega_{k}(k \geqq 0)$, they also introduced and studied the corresponding class $k-\mathcal{S} \mathcal{T}$ of $k$-starlike functions (see Definition 3 below).

Moreover, for fixed $k, \Omega_{k}$ represents the conic region bounded successively by the imaginary axis for $(k=0)$, for $k=1$ a parabola, for $0<k<1$ the right branch of a hyperbola, and for $k>1$ an ellipse. For these conic regions, the following functions $p_{k}(z)$, which are given by (3), play the role of extremal functions.

$$
p_{k}(z)= \begin{cases}\frac{1+z}{1-z}=1+2 z+2 z^{2}+\cdots & (k=0) \\ 1+\frac{2}{\pi^{2}}\left(\log \frac{1+\sqrt{z}}{1-\sqrt{z}}\right)^{2} & (k=1) \\ 1+\frac{2}{1-k^{2}} \sinh ^{2}\left\{\left(\frac{2}{\pi} \arccos k\right) \arctan (h \sqrt{z})\right\} & (0 \leqq k<1) \\ 1+\frac{1}{k^{2}-1} \sin \left(\frac{\pi}{2 K(\kappa)} \int_{0}^{\frac{u(z)}{\sqrt{\kappa}}} \frac{d t}{\sqrt{1-t^{2}} \sqrt{1-\kappa^{2} t^{2}}}\right)+\frac{1}{k^{2}-1} & (k>1),\end{cases}
$$

where:

$$
u(z)=\frac{z-\sqrt{\kappa}}{1-\sqrt{\kappa} z} \quad(\forall z \in \mathbb{E})
$$

and $\kappa \in(0,1)$ is chosen such that:

$$
k=\cosh \left(\frac{\pi K^{\prime}(\kappa)}{4 K(\kappa)}\right) .
$$

Here, $K(\kappa)$ is Legendre's complete elliptic integral of the first kind and: 


$$
K^{\prime}(\kappa)=K\left(\sqrt{1-\kappa^{2}}\right),
$$

that is, $K^{\prime}(\kappa)$ is the complementary integral of $K(\kappa)$.

These conic regions are being studied and generalized by several authors (see, for example, [10-13]).

The class $k-\mathcal{S} \mathcal{T}$ is defined as follows.

Definition 3. A function $f \in \mathcal{A}$ is said to be in the class $k-\mathcal{S} \mathcal{T}$ if and only if:

$$
\frac{z f^{\prime}(z)}{f(z)} \prec p_{k}(z) \quad(\forall z \in \mathbb{E} ; k \geqq 0)
$$

or, equivalently,

$$
\Re\left(\frac{z f^{\prime}(z)}{f(z)}\right)>k\left|\frac{z f^{\prime}(z)}{f(z)}-1\right|
$$

The class of $k$-uniformly close-to-convex functions denoted by $k-\mathcal{U K}$ was studied by Acu [14].

Definition 4. A function $f \in \mathcal{A}$ is said to be in the class $k-\mathcal{U K}$ if and only if:

$$
\Re\left(\frac{z f^{\prime}(z)}{g(z)}\right)>k\left|\frac{z f^{\prime}(z)}{g(z)}-1\right|,
$$

where $g \in k-\mathcal{S} \mathcal{T}$.

In recent years, several interesting subclasses of analytic functions were introduced and investigated from different viewpoints (see, for example, [6,15-20]; see also [21-25]). Motivated and inspired by the recent and current research in the above-mentioned work, we here introduce and investigate certain new subclasses of analytic and $p$-valent functions by using the concept of conic domains and spiral-like functions as follows.

Definition 5. Let $f \in \mathcal{A}(p)$. Then, $f \in k-\mathcal{K}(p, \lambda)$ for a real number $\lambda$ with $|\lambda|<\frac{\pi}{2}$ if and only if:

$$
\Re\left(\frac{e^{i \lambda}}{p} \frac{z f^{\prime}(z)}{\psi(z)}\right)>k\left|\frac{z f^{\prime}(z)}{\psi(z)}-p\right|+\rho \cos \lambda \quad(k \geqq 0 ; 0 \leqq \rho<1)
$$

for some $\psi \in \mathcal{S}^{*}$.

Definition 6. Let $f \in \mathcal{A}(p)$. Then, $f \in k-\mathcal{Q}(p, \lambda)$ for a real $\lambda$ with $|\lambda|<\frac{\pi}{2}$ if and only if:

$$
\Re\left(\frac{e^{i \lambda}}{p} \frac{z f^{\prime}(z)}{\psi^{\prime}(z)}\right)>k\left|\frac{\left(z f^{\prime}(z)\right)^{\prime}}{\psi^{\prime}(z)}-p\right|+\rho \cos \lambda \quad(k \geqq 0 ; 0 \leqq \rho<1)
$$

for some $\psi \in \mathcal{C}$.

Definition 7. Let $f \in \mathcal{A}(p)$ with:

$$
\frac{f^{\prime}(z) f(z)}{p z} \neq 0
$$

and for some real $\phi$ and $\lambda$ with $|\lambda|<\frac{\pi}{2}$. Then, $f \in k-\mathcal{Q}(\phi, \lambda, \eta, f, \psi)$ if and only if:

$$
\Re(\mathcal{M}(\phi, \lambda, \eta, f, \psi))>k|\mathcal{M}(\phi, \lambda, \eta, f, \psi)-p|+\rho \cos \lambda,
$$


where

$$
\begin{aligned}
\mathcal{M}(\phi, \lambda, \eta, f, \psi)=\left(e^{i \lambda}-\phi \cos \lambda\right) \frac{z f^{\prime}(z)}{p \psi(z)} & \\
& +\frac{\phi \cos \lambda}{p-\eta}\left(\frac{\left(z f^{\prime}(z)\right)^{\prime}}{\psi^{\prime}(z)}-\eta\right) \quad\left(\frac{-1}{2} \leqq \eta<1\right) .
\end{aligned}
$$

\section{A Set of Lemmas}

Each of the following lemmas will be needed in our present investigation.

Lemma 1. (see [26] p. 70) Let $h$ be a convex function in $\mathbb{E}$ and:

$$
q: \mathbb{E} \Longrightarrow \mathbb{C} \text { and } \Re(q(z))>0 \quad(z \in \mathbb{E}) .
$$

If $p$ is analytic in $\mathbb{E}$ with:

$$
p(0)=h(0)
$$

then:

$$
p(z)+q(z) z p^{\prime}(z) \prec h(z) \text { implies } p(z) \prec h(z) \text {. }
$$

Lemma 2. (see [26] p. 195) Let $h$ be a convex function in $\mathbb{E}$ with:

$$
h(0)=0 \text { and } A>1 .
$$

Suppose that $j \geqq \frac{4}{h^{\prime}(0)}$ and that the functions $B(z), C(z)$ and $D(z)$ are analytic in $\mathbb{E}$ and satisfy the following inequalities:

$$
\Re\{B(z)\} \geqq A+|C(z)-1|-\Re(C(z)-1)+j D(z), \quad z \in \mathbb{E} .
$$

If $p$ is analytic in $\mathbb{E}$ with:

$$
p(z)=1+a_{1} z+a_{2} z^{2}+\cdots
$$

and the following subordination relation holds true:

$$
A z^{2} p^{\prime \prime}(z)+B(z) z p^{\prime}(z)+C(z) p(z)+D(z) \prec h(z),
$$

then:

$$
p(z) \prec h(z) .
$$

\section{Main Results and Their Demonstrations}

In this section, we will prove our main results.

Theorem 1. A function $f \in \mathcal{A}$ is in the class $k-\mathcal{Q}(\phi, \lambda, \eta, f, \psi)$ if:

$$
\sum_{n=2}^{\infty} \ddot{U}_{n}(p, \phi, \lambda, \eta, \xi)<p^{2}(p-\eta)
$$

where:

$$
\begin{aligned}
\ddot{U}_{n}(p, \phi, \lambda, \eta, \xi)= & (k+1)\left[\left(e^{i \lambda}-\phi \cos \lambda\right)(p-\eta) p+p^{4} \phi \cos \lambda\right. \\
& +\left(e^{i \lambda}-\phi \cos \lambda\right)(p-\eta)(n+p)\left|a_{n+p}\right|+(n+p)^{2}\left|a_{n+p}\right| \\
& +\left[\left(n p \phi \cos \lambda+p^{3}(p-\eta)\right](n+p)\left|b_{n+p}\right|+n p^{2} \phi \cos \lambda-p^{3}(p-\eta) .\right.
\end{aligned}
$$


Proof. Let us assume that the relation (4) holds true. It now suffices to show that:

$$
k|\mathcal{M}(\phi, \lambda, \eta, f, \psi)-p|-\Re|\mathcal{M}(\phi, \lambda, \eta, f, \psi)-p|<1
$$

We first consider:

$$
\begin{aligned}
|\mathcal{M}(\phi, \lambda, \eta, f, \psi)-p| & \left|\left(e^{i \lambda}-\phi \cos \lambda\right) \frac{z f^{\prime}(z)}{p \psi(z)}+\frac{\phi \cos \lambda}{(p-\eta)}\left(\frac{\left(z f^{\prime}(z)\right)^{\prime}}{\psi^{\prime}(z)}-\eta\right)-p\right| \\
= & \mid \frac{\left(e^{i \lambda}-\phi \cos \lambda\right)(p-\eta) f^{\prime}(z)}{p(p-\eta) \psi^{\prime}(z)}+\frac{p \phi \cos \lambda\left(z f^{\prime}(z)\right)^{\prime}}{p(p-\eta) \psi^{\prime}(z)}- \\
& -\frac{\eta p \phi \cos \lambda \psi^{\prime}(z)}{p(p-\eta) \psi^{\prime}(z)}-\frac{p^{2}(p-\eta) \psi^{\prime}(z)}{p(p-\eta) \psi^{\prime}(z)} \mid .
\end{aligned}
$$

Now, by using the series form of the functions $f$ and $\psi$ given by:

$$
f(z)=z^{p}+\sum_{n=2}^{\infty} a_{n+p} z^{n+p}
$$

and:

$$
\psi(z)=z^{p}+\sum_{n=2}^{\infty} b_{n+p} z^{n+p}
$$

in the above relation, we have:

$$
\begin{aligned}
&|\mathcal{M}(\phi, \lambda, \eta, f, \psi)-p| \\
&=\mid \frac{\left(e^{i \lambda}-\phi \cos \lambda\right)(p-\eta)\left(p z^{p-1}\right)+p \phi \cos \lambda\left(p^{2} z^{p-1}\right)}{p(p-\eta)\left(p z^{p-1}+\sum_{n=2}^{\infty}(n+p) b_{n+p} z^{n+p-1}\right)} \\
& \quad+\frac{\sum_{n=2}^{\infty}(n+p) a_{n+p} z^{n+p-1}\left[\left(e^{i \lambda}-\phi \cos \lambda\right)(p-\eta)+(n+p)\right]}{p(p-\eta)\left(p z^{p-1}+\sum_{n=2}^{\infty}(n+p) b_{n+p} z^{n+p-1}\right)}-\frac{n \phi \cos \lambda}{(p-\eta)}-p \mid \\
& \leqq \frac{\left(e^{i \lambda}-\phi \cos \lambda\right)(p-\eta)(p)+p \phi \cos \lambda\left(p^{2}\right)}{p(p-\eta)\left(p+\sum_{n=2}^{\infty}(n+p)\left|b_{n+p}\right|\right)} \\
& \quad+\frac{\sum_{n=2}^{\infty}(n+p)\left|a_{n+p}\right|\left\{\left(e^{i \lambda}-\phi \cos \lambda\right)(p-\eta)+(n+p)\right\}}{p(p-\eta)\left(p+\sum_{n=2}^{\infty}(n+p)\left|b_{n+p}\right|\right)}-\left\{\frac{n \phi \cos \lambda}{(p-\eta)}+p\right\} .
\end{aligned}
$$

We now see that:

$$
\begin{aligned}
k|\mathcal{M}(\phi, \lambda, \eta, f, \psi)-p|-\Re\{\mathcal{M}(\phi, \lambda, \eta, f, \psi)-p\} & \leqq(k+1)|\mathcal{M}(\phi, \lambda, \eta, f, \psi)-p| \\
\leqq & \leqq\left[( k + 1 ) \left[\frac{\left(e^{i \lambda}-\phi \cos \lambda\right)(p-\eta)(p)+p \phi \cos \lambda\left(p^{2}\right)}{p(p-\eta)\left(p+\sum_{n=2}^{\infty}(n+p)\left|b_{n+p}\right|\right)}\right.\right. \\
& \left.+\frac{\sum_{n=2}^{\infty}(n+p)\left|a_{n+p}\right|\left[\left(e^{i \lambda}-\phi \cos \lambda\right)(p-\eta)+(n+p)\right]}{p(p-\eta)\left(p+\sum_{n=2}^{\infty}(n+p)\left|b_{n+p}\right|\right)}-\left[\frac{n \phi \cos \lambda}{(p-\eta)}+p\right]\right] .
\end{aligned}
$$


The above inequality is bounded above by one, if:

$$
\begin{aligned}
(k+1) & {\left[\left(e^{i \lambda}-\phi \cos \lambda\right)(p-\eta) p\right]+(p \phi \cos \lambda) p^{2} } \\
& +\left(\sum_{n=2}^{\infty}(n+p)\left|a_{n+p}\right|\right)\left\{\left(e^{i \lambda}-\phi \cos \lambda\right)(p-\eta)+(n+p)\right\}-\left[\frac{n \phi \cos \lambda}{(p-\eta)}-p\right] \\
& \cdot\left\{p(p-\eta)\left(p+\sum_{n=2}^{\infty}(n+p)\left|b_{n+p}\right|\right)\right\} \\
\leqq & p(p-\eta) p+\sum_{n=2}^{\infty}(n+p)\left|b_{n+p}\right| .
\end{aligned}
$$

Hence:

$$
\sum_{n=2}^{\infty} \ddot{U}_{n}(p, \phi, \lambda, \eta, \xi) \leqq p^{2}(p-\eta)
$$

where $\ddot{U}_{n}(p, \phi, \lambda, \eta, \xi)$ is given by (5), which completes the proof of Theorem 1 .

Theorem 2. A function $f \in \mathcal{A}(p)$ satisfies the condition:

$$
\left|\frac{1}{e^{i j} F(z)}-\frac{1}{2 \rho}\right|<\frac{1}{2 \rho} \quad(0 \leqq \rho<1 ; j \in \mathbb{R})
$$

if and only if $f \in 0-\mathcal{K}(p, \lambda)$, where

$$
F(z)=\frac{z f^{\prime}(z)}{p \psi(z)}
$$

Proof. Suppose that $f$ satisfies (7). We then can write:

$$
\begin{aligned}
\left|\frac{2 \rho-e^{i j} F(z)}{e^{i j} F(z) 2 \rho}\right| & <\frac{1}{2 \rho} \\
& \Longleftrightarrow\left(\left|\frac{2 \rho-e^{i j} F(z)}{e^{i j} F(z) 2 \rho}\right|\right)^{2}<\left(\frac{1}{2 \rho}\right)^{2} \\
& \Longleftrightarrow\left(2 \rho-e^{i j} F(z)\right)\left(\overline{2 \rho-e^{i j} F(z)}\right)<e^{-i j} \overline{F(z)} e^{i j} F(z) \\
& \Longleftrightarrow 4 \rho^{2}-2 \rho\left[e^{-i j} \overline{F(z)}+e^{i j} F(z)\right]+F(z) \overline{F(z)}<F(z) \overline{F(z)} \\
& \Longleftrightarrow 4 \rho^{2}-2 \rho\left[e^{-i j} \overline{F(z)}+e^{i j} F(z)\right]<0 \\
& \Longleftrightarrow 2 \rho-2 \Re\left[e^{i j} \overline{F(z)}\right]<0 \\
& \Longleftrightarrow \Re\left[e^{i j} F(z)\right]>\rho \\
& \Longleftrightarrow \Re\left(e^{i j} \frac{z f^{\prime}(z)}{p \psi(z)}\right)>\rho .
\end{aligned}
$$

This completes the proof of Theorem 2.

Theorem 3. For $0 \leqq \varphi_{1}<\varphi_{2}$, it is asserted that:

$$
k-\mathcal{Q}\left(p, \varphi_{2}, \lambda, \eta\right) \subset 0-\mathcal{Q}\left(p, \varphi_{1}, \lambda, \eta\right)
$$


Proof. Let $f(z) \in k-\mathcal{Q}\left(p, \varphi_{2}, \lambda, \eta\right)$. Then:

$$
\begin{aligned}
\frac{1}{p-\eta} & {\left[\left(e^{i \lambda}-\phi_{1} \cos \lambda\right)(p-\eta) \frac{z f^{\prime}(z)}{p \psi(z)}+\varphi_{1} \cos \lambda\left(\frac{\left(z f^{\prime}(z)\right)^{\prime}}{\psi(z)^{\prime}}-\eta\right)\right] } \\
= & \frac{\varphi_{1}}{\varphi_{2}}\left[\left(e^{i \lambda}-\varphi_{2} \cos \lambda\right) \frac{z f^{\prime}(z)}{p \psi(z)}+\frac{\varphi_{2} \cos \lambda}{(p-\eta)}\left(\frac{\left(z f^{\prime}(z)\right)^{\prime}}{p \psi(z)^{\prime}}-\eta\right)\right] \\
& \quad-\left(\frac{\varphi_{1}-\varphi_{2}}{\varphi_{2}}\right) e^{i \lambda} \frac{s f^{\prime}(z)}{p \psi(z)} \\
= & \frac{\varphi_{1}}{\varphi_{2}} H_{1}(z)+\left(1-\frac{\varphi_{1}}{\varphi_{2}}\right) H_{2}(z)=H(z),
\end{aligned}
$$

where:

$$
H_{1}(z)=\left(e^{i \lambda}-\varphi_{2} \cos \lambda\right) \frac{z f^{\prime}(z)}{p \psi(z)}+\frac{\varphi_{2} \cos \lambda}{(p-\eta)}\left(\frac{\left(z f^{\prime} z\right)^{\prime}}{\psi^{\prime}(z)}-\eta\right) \in \mathcal{P}\left(h_{k, \rho}\right) \subset \mathcal{P}(\rho)
$$

and:

$$
H_{2}(z)=e^{i \lambda} \frac{z f^{\prime}(z)}{p \psi(z)} \in \mathcal{P}(\rho) .
$$

Since $\mathcal{P}(\rho)$ is a convex set (see [27]), we therefore have $H(z) \in \mathcal{P}(\rho)$. This implies that $f \in$ $0-\mathcal{Q}\left(p, \varphi_{1}, \lambda, \eta\right)$. Thus:

$$
k-\mathcal{Q}\left(p, \varphi_{2}, \lambda, \eta\right) \subset 0-\mathcal{Q}\left(p, \varphi_{1}, \lambda, \eta\right) .
$$

The proof of Theorem 3 is now completed.

Theorem 4. Let $\phi>0$ and $\lambda<\frac{\pi}{2}$. Then:

$$
k-\mathcal{Q}(p, \phi, \lambda, \eta, \xi) \subset k-\mathcal{K}(p, 0, \xi)
$$

Proof. Let $f \in k-\mathcal{Q}(p, \phi, \lambda, \eta, \xi)$, and suppose that:

$$
\frac{f^{\prime}(z)}{\psi^{\prime}(z)}=p(z),
$$

where $p(z)$ is analytic and $p(0)=1$. Now, by differentiating both sides of (8) with respect to $z$, we have:

$$
\frac{\left(z f^{\prime}(z)\right)^{\prime}}{\psi^{\prime}(z)}=z p^{\prime}(z)+p(z) \varepsilon(z),
$$

where:

$$
\varepsilon(z)=\frac{\left(z \psi^{\prime}(z)\right)^{\prime}}{\psi^{\prime}(z)} .
$$

By using (8) and (9) in (4), we arrive at:

$$
\begin{aligned}
\mathcal{M}(\phi, \lambda, \eta, f, \psi) & =\left(e^{i \lambda}-\phi \cos \lambda\right) \frac{p(z)}{p}+\frac{\phi \cos \lambda}{p-\eta}\left(z p^{\prime}(z)+p(z) \varepsilon(z)-\eta\right) \\
& =\frac{\phi \cos \lambda}{p-\eta} z p^{\prime}(z)+\left(\frac{e^{i \lambda}}{p}-\frac{\phi \cos \lambda}{p-\varepsilon(z)}\right)\left(\frac{\phi \cos \lambda}{p-\eta}\right) p(z)-\frac{\eta \phi \cos \lambda}{p-\eta} \\
& =B(z) z p^{\prime}(z)+C(z) p(z)+D(z),
\end{aligned}
$$

where:

$$
B(z)=\frac{\phi \cos \lambda}{p-\eta}
$$




$$
C(z)=\frac{e^{i \lambda}(p-\eta)-\phi \cos \lambda(p-\eta)+\phi \cos \lambda \varepsilon(z) p}{p(p-\eta)}
$$

and:

$$
D(z)=\frac{\eta \phi \cos \lambda}{p-\eta}
$$

Now, since $f \in k-\mathcal{Q}(p, \phi, \lambda, \eta, \xi)$, we have:

$$
B(z) z p^{\prime}(z)+C(z) p(z)+D(z) \prec p_{k}(z),
$$

which, upon replacing $p(z)$ by:

$$
p_{*}(z)=p(z)-1,
$$

and $p_{k}(z)$ by:

$$
p_{k}^{*}(z)=p_{k}(z)-1,
$$

shows that the above subordination in (11) becomes as follows:

$$
B(z) z p_{x}^{\prime}(z)+C(z) p_{x}(z)+D_{*}(z) \prec p_{k}^{*}(z)
$$

where:

$$
D_{*}(z)=C(z)+D(z)-1 \text {. }
$$

We now apply Lemma 2 with:

$$
A=0
$$

and

$$
p_{*}(z) \prec p_{k}^{*}(z)
$$

We thus find that:

$$
\frac{f^{\prime}(z)}{\psi^{\prime}(z)}=p(z) \prec p_{k}^{*}(z) .
$$

This complete the proof of Theorem 4.

For $f \in \mathcal{A}$, we next consider the integral operator defined by:

$$
F(z)=I_{m}[f]=\frac{m+1}{z^{m}} \int_{0}^{z} t^{m-1} f(t) d t .
$$

This operator was given by Bernardi [28] in the year 1969. In particular, the operator $I_{1}$ was considered by Libera [29]. We prove the following result.

Theorem 5. Let $f(z) \in k-\mathcal{Q}(p, \phi, \lambda, \eta, \xi)$. Then, $I_{m}[f] \in \mathcal{K}(p, 0, \xi)$.

Proof. Let the function $\psi(z)$ be such that:

$$
\mathcal{M}(\phi, \lambda, \eta, f, \psi)=\left(e^{i \lambda}-\phi \cos \lambda\right) \frac{z f^{\prime}(z)}{p \psi(z)}+\frac{\phi \cos \lambda}{(p-\eta)}\left(\frac{\left(z f^{\prime}(z)\right)^{\prime}}{\psi^{\prime}(z)}-\eta\right) .
$$

Then, according to [14], the function $G=I_{m}[f] \in \mathcal{C D}(k, \delta)$. Furthermore, from (14), we deduce that:

$$
(1+m) f(z)=(1+m) F(z)+z(F(z))^{\prime}
$$

and:

$$
(1+m) g(z)=(1+m) G(z)+z(G(z))^{\prime} .
$$


If we now put:

$$
p(z)=\frac{F^{\prime}(z)}{G^{\prime}(z)}
$$

and:

$$
q(z)=\frac{1}{(m+1)+\left(\frac{z G^{\prime \prime}(z)}{G^{\prime}(z)}\right)},
$$

then, by simple computations, we find that:

$$
\frac{f(z)}{\psi(z)}=\frac{(1+m) F^{\prime}(z)+z F^{\prime \prime}(z)}{(1+m) G^{\prime}(z)+z G^{\prime \prime}(z)}
$$

or, equivalently, that:

$$
\frac{f^{\prime}(z)}{\psi^{\prime}(z)}=p(z)+z p^{\prime}(z) q(z)
$$

We now let:

$$
\frac{f^{\prime}(z)}{\psi^{\prime}(z)}=p(z)+z p^{\prime}(z) q(z)=h(z)
$$

where the function $h(z)$ is analytic in $\mathbb{E}$ with $h(0)=1$. Then, by using (18), we have:

$$
\frac{(z f(z))^{\prime}}{\psi^{\prime}(z)}=z h^{\prime}(z)+\varepsilon(z) h(z)
$$

where:

$$
\varepsilon(z)=\frac{\left(z \psi^{\prime}(z)\right)^{\prime}}{\psi^{\prime}(z)} .
$$

Furthermore, by using (18) and (19) in (4), we obtain:

$$
\begin{aligned}
\mathcal{M}(\alpha, \beta, \gamma, \lambda, \delta, f) & =\left(e^{i \lambda}-\theta \cos \lambda\right) \frac{z f^{\prime}(z)}{\psi^{\prime}(z)}+\frac{\phi \cos \lambda}{p-\eta}\left(\frac{\left(z f^{\prime}(z)\right)^{\prime}}{\psi^{\prime}(z)}-\eta\right) \\
& =\left(e^{i \lambda}-\theta \cos \lambda\right)+\frac{\phi \cos \lambda}{p-\eta} z h^{\prime}(z)+\left[z h^{\prime}(z)+\varepsilon(z) h(z)-\eta\right] \\
& =\frac{\phi \cos \lambda}{p-\eta} z h^{\prime}(z)+\left(e^{i \lambda}-\phi \cos \lambda+\frac{\phi \cos \lambda}{p-\eta}\right) h(z)-\frac{\eta(\phi \cos \lambda)}{p-\eta} \\
& =B(z) z h^{\prime}(z)+C(z) h(z)+D(z),
\end{aligned}
$$

where:

$$
\begin{gathered}
B(z)=\frac{\phi \cos \lambda}{p-\eta} \\
C(z)=\frac{\left((p-\eta) e^{i \lambda}-(p-\eta) \phi \cos \lambda+\phi \cos \lambda\right)}{p-\eta}
\end{gathered}
$$

and:

$$
D(z)=\frac{\eta(\phi \cos \lambda)}{p-\eta}
$$

Now, if we apply Lemma 1 with $A=0$, we get:

$$
\frac{f^{\prime}(z)}{\psi^{\prime}(z)}=h(z) \prec p_{k}(z) .
$$

Furthermore, from (18), we have:

$$
p(z)+z p^{\prime}(z) q(z) \prec p_{k}(z) .
$$


By using Lemma 2 on (20), we obtain the desired result. This completes the proof of Theorem 5.

\section{Conclusions}

Using the idea of spiral-like and close-to-convex functions, we have introduced Mocanu-type functions associated with conic domains. We have derived some interesting results such as sufficiency criteria, inclusion results, and integral-preserving properties. We have also proven that the our newly-defined function classes are closed under the famous Libera operator.

Author Contributions: conceptualization, H.M.S. and Q.Z.A.; methodology, N.K.; software, M.T.R. and M.D.; validation, H.M.S., M.D. and Y.Z.; formal analysis, H.M.S. and Q.Z.A; investigation, M.D. and M.T.R.; writing-original draft preparation, H.M.S.; and Y.Z writing-review and editing, N.K. and M.D.; visualization, M.T.R.; supervision, H.M.S.; funding acquisition, M.D.

Funding: The third author is partially supported by UKM grant: GUP-2017-064.

Conflicts of Interest: The authors declare that they have no competing interests.

\section{References}

1. Duren, P.L. Univalent Functions, Grundlehren der Mathematischen Wissenschaften, Band 259; Springer-Verlag: New York, NY, USA; Berlin/Heidelberg, Germany; Tokyo, Japan, 1983.

2. Spaček, L. Prispevek $k$ teorii funkei prostych. Casǒpis. Pest. Mat. 1933, 62, 12-19.

3. Libera, R. J. Univalent $a$-spiral functions, Can. J. Math. 1967, 19, 449-456.

4. Silvia, M.E. On a subclass of spiral-like functions, Proc. Am. Math. Soc. 1974, 44, 411-420. [CrossRef]

5. Umarani, P. On a subclass of spiral-like functions, Indian J. Pure Appl. Math. 1979, 10, 1292-1297.

6. Noor, K.I.; Khan, N.; Ahmad, Q.Z. Some properties of multivalent spiral-like functions. Maejo Int. J. Sci. Technol. 2018, 3, 353-364.

7. Kanas. S.; Wiśniowska, A. Conic regions and $k$-uniform convexity. J. Comput. Appl. Math. 1999, 105, 327-336. [CrossRef]

8. Kanas. S.; Wiśniowska, A. Conic domains and starlike functions. Rev. Roum. Math. Pures Appl. 2000, $45,647-657$.

9. Kanas, S.; Srivastava, H. M.; Linear operators associated with $k$-uniformly convex functions. Integral Transforms Spec. Funct. 2000, 9, 121-132. [CrossRef]

10. Kanas, S.; Răducanu, D. Some class of analytic functions related to conic domains. Math. Slovaca 2014, 64, 1183-1196. [CrossRef]

11. Noor, K.I.; Malik, S.N. On coefficient inequalities of functions associated with conic domains. Comput. Math. Appl. 2011, 62, 2209-2217. [CrossRef]

12. Shams, S.; Kulkarni, S.R.; Jahangiri, J.M. Classes of uniformly starlike and convex functions. Int. J. Math. Math. Sci. 2004, 55, 2959-2961. [CrossRef]

13. Srivastava, H.M.; Shanmugam, T.N.; Ramachandran, C.; Sivassurbramanian, S. A new subclass of $k$-uniformly convex functions with negative coefficients. J. Inequal. Pure Appl. Math. 2007, 8, 1-14.

14. Acu, M. On a subclass of $k$-uniformly close to convex functions. Gen. Math. 2006, 14, 55-64.

15. Arif, M.; Dziok, J.; Raza, M.; Sokól, J. On products of multivalent close-to-star functions, J. Inequal. Appl. 2015, 2015, 1-14. [CrossRef]

16. Khan, N.; Khan, B.; Ahmad, Q.Z; Ahmad, S. Some Convolution properties of multivalent analytic functions. AIMS Math. 2017, 2, 260-268. [CrossRef]

17. Noor, K.I.; Khan, N.; Noor, M.A. On generalized spiral-like analytic functions. Filomat 2014, 28, 1493-1503. [CrossRef]

18. Raza, M.; Din, M.U.; Malik, S.N. Certain geometric properties of normalized Wright functions. J. Funct. Spaces 2016, 2016. [CrossRef]

19. Srivastava, H.M.; Eker, S.S. Some applications of a subordination theorem for a class of analytic functions. Appl. Math. Lett. 2008, 21, 394-399. [CrossRef]

20. Srivastava, H.M.; El-Ashwah, R.M.; Breaz, N. A certain subclass of multivalent functions involving higher-order derivatives. Filomat 2016, 30, 113-124. [CrossRef] 
21. Aldweby, H.; Darus, M. A note on $q$-integral operators. Electron. Notes Discret. Math. 2018, 67, 25-30. [CrossRef]

22. Aldweby, H.; Darus, M. On Fekete-Szegö problems for certain subclasses defined by $q$-derivative. J. Funct. Spaces 2017, 1-5. . [CrossRef]

23. Elhaddad, S.; Aldweby, H.; Darus, M. Some Properties on a class of harmonic univalent functions defined by q-analoque of Ruscheweyh operator. J. Math. Anal. 2018, 9, 28-35.

24. Hussain, S.; Khan, S.; Zaighum, M.A.; Darus, M. Applications of a $q$-Sălăgean type operator on multivalent functions. J. Inequal. Appl. 2018, 301,1-12. [CrossRef]

25. Rasheed, A.; Hussain, S.; Zaighum, M.A.; Darus, M. Class of analytic function related with uniformly convex and Janowski's functions. J. Funct. Spaces 2018, 2018. [CrossRef]

26. Miller, S.S.; Mocanu, P.T. Differential Subordinations: Theory and Applications; Marcel Dekker: New York, NY, USA, 2000.

27. Kanas. S. Techniques of the differential subordination for domains bounded by conic sections. Int. J. Math. Math. Sci. 2003, 38, 2389-2400. [CrossRef]

28. Bernardi, S.D. Convex and starlike univalent functions. Trans. Am. Math. Soc. 1969, 135, 429-446. [CrossRef]

29. Libera, R.J. Some classes of regular univalent functions. Proc. Am. Math. Soc. 1965, 16, 755-758. [CrossRef]

(C) 2019 by the authors. Licensee MDPI, Basel, Switzerland. This article is an open access article distributed under the terms and conditions of the Creative Commons Attribution (CC BY) license (http://creativecommons.org/licenses/by/4.0/). 